\title{
Las Desigualdades de Heisenberg. Interpretación y didáctica
}

\author{
Rafael Andrés Alemañ Berenguer \\ raalbe.autor@gmail.com \\ Universidad Miguel Hernández \\ Elche-España
}

\section{Resumen.}

La importancia primordial del inadecuadamente denominado "Principio de Heisenberg" en la física cuántica, no ha evitado una gran cantidad de malentendidos sobre el mismo. Para caracterizar tales malentendidos, 25 profesores de física y química de enseñanza media fueron sometidos a un breve cuestionario escrito. Ninguno de ellos ofreció una respuesta correcta, y sus contestaciones revelaron que los errores surgen de su confusión sobre los fundamentos de la física cuántica así como la utilización de estrategias de aprendizaje equivocadas.

Palabras claves: Heisenberg, incertidumbre, indeterminación, cuántica, conmutador

\begin{abstract}
.
The fundamental importance of the inadequately denominated "Principle of Heisenber" in quantum physics, has not avoided a great amount of mistakes on it. In order to characterize such mistakes, 25 high school teachers of physics and chemistry were asked to answer a brief written test. None of them offered a correct answer, and their comments revealed that the errors arise from their confusion on the proper foundations of quantum physics as well as the use of mistaken strategies of learning.
\end{abstract}

KeyWords: Heisenberg, uncertainty, indetermination, quantum, commutator

\subsection{Introducción.}

Uno de los más activos campos de investigación en la didáctica de las ciencias es el referido a la existencia de errores conceptuales entre los estudiantes (Gustone, 1988) y a su resistencia (Osborne y Wittrock, 1983) ante el cambio conceptual que caracteriza un adecuado aprendizaje significativo desde la óptica constructivista. Este estudio no se ha limitado únicamente a examinar los prejuicios conceptuales de los estudiantes, sino que ha sido extendido a la investigación de las confusiones de los propios profesores (Baird, 1988) con muy interesantes resultados. Los diversos trabajos llevados a efecto en este campo de investigación han intentado dar respuesta a diferentes preguntas relacionadas con el proceso de aprendizaje, como pueden ser: cuáles son los errores o dificultades en cuestión, cuál puede ser su origen, en qué grado se hallan extendidos, por qué son 
tan resistentes al cambio conceptual, y cómo deben ser afrontados para evitarlos o superarlos. De acuerdo con ello, en el presente trabajo se intentará dar respuesta a algunas de estas preguntas en torno a los errores de interpretación, muy ampliamente extendidos, sobre las desigualdades de Heisenberg en física cuántica.

Las nociones fundamentales de la teoría cuántica se han convertido en pieza clave dentro de los diseños curriculares impulsados por diversas reformas pedagógicas. Semejante relevancia no resulta en absoluto inmerecida, pues son innumerables los ejemplos de aplicaciones tecnológicas y avances científicos basados en esta rama de la microfísica. El efecto fotoeléctrico, las técnicas espectroscópicas, el efecto Compton, la tabla periódica química, la estabilidad de la materia, las propiedades eléctricas y magnéticas de los sólidos cristalinos, la superconductividad y la superfluidez, la fusión y fisión nuclear, los aceleradores de partículas y la misma estabilidad de la materia, son dispositivos y fenómenos que deben a la física cuántica su existencia o su correcta explicación.

\subsection{Las desigualdades de Heisenberg}

En el marco de la física cuántica, uno de los resultados más célebres resultados - que en ocasiones se toma como representativo de la teoría - es el de unas fórmulas matemáticas obtenidas por el alemán Werner Heisenberg entre 1925 y 1927. Su significado físico sigue siendo hoy día casi tan controvertido como en los tiempos de su publicación, y en ello no ha tenido poco que ver la funesta costumbre de usar los conceptos clásicos de partícula y onda en el contexto no clásico de la teoría cuántica, como señaló ese mismo autor (Heisenberg, 1930). Como en cualquier teoría científica, la correcta interpretación del formalismo cuántico ha de rastrearse hasta el proceso deductivo que permite obtener los enunciados en discusión. En el caso que nos ocupa, supongamos que $p_{i}$ y $q_{i}$ (con $i=1,23$ ) designan respectivamente las componentes del momento y la posición de un objeto físico, como un electrón, y que $\psi$ representa su estado físico en un instante dado. Denotemos por $\Delta_{\psi} p_{i}$ y $\Delta_{\psi} q_{i}$ las desviaciones típicas correspondientes a ambas magnitudes. Las desigualdades de Heisenberg, así pues, son:

$$
\Delta_{\psi} p_{i} \cdot \Delta_{\psi} q_{i} \geq(h / 4 \pi) \delta_{i j}
$$

donde $h$ es la constante de Planck y $\delta_{i j}$ es la delta de $\operatorname{Kronecker}\left(\delta_{i j}=1\right.$ si $i=j$, y $\delta_{i j}=0$ si $\left.i \neq j\right)$ en la que $i, j=1,2,3$.

De la anterior deducción se desprende que todo el razonamiento se ha ceñido únicamente a las componentes espaciales de las variables canónicas. Contra la opinión usual, estas desigualdades no rigen sobre las cuartas componentes de ambas variables dinámicas espacio-temporales, la referida al tiempo (en el caso de $q$ ) y la correspondiente a la energía (para $p$ ). En realidad el parámetro $t$ del tiempo, es lo que Dirac llamaba un número $c$ (una magnitud clásica), y por tanto carece de dispersión para todos los estados posibles, $\Delta_{\psi} t=0$ para todo $\Delta$ (Bunge, 1970).

Las desigualdades de Heisenberg, por otra parte, resultan completamente generales en relación con cualquier entidad cuántica en cualquier estado, y su validez se mantiene tanto si ésta se halla bajo observación como si no. Tal cual se acaba de ver, en la exposición precedente no hemos mencionado ni el tipo de entidad, ni la forma de observar o de medir, y ni tan siquiera se supone un hamiltoniano específico.

Como consecuencia de lo anterior, se deduce que los nombres habitualmente asignados a estas desigualdades - "principio de incertidumbre" o "principio de indeterminación" - son incorrectos. En este sentido, resultan 
falaces las tentativas de obtener estas fórmulas mediante el análisis de situaciones experimentales tanto reales como ideales, método este tan socorrido en multitud de libros de text $\mathrm{c}^{1}$. Son erróneas, por consiguiente, tanto las analogías ópticas - véase el típico ejemplo de la difracción de la luz por una ranura (Wichman, 1991; Levine, 1977) - como la del microscopio de rayos gamma de Heisenberg (Alonso-Finn, 1971; Dicke-Wittke, 1975; Gassiorowicz, 1974).

El caso de los símiles ópticos es particularmente desorientador por cuanto emplea proposiciones que no pertenecen a la teoría cuántica (por ejemplo, la fórmula del poder de resolución de una lente, o la que proporciona la relación inversa entre la amplitud de banda y la anchura espacial de un paquete de ondas), referentes a sistemas macrofísicos formados por una ingente cantidad de fotones con distintas longitudes de onda. Todas estas consideraciones, puramente analógicas y heurísticas, se infiltraron en los libros de texto con una pretensión didáctica inicial tan poderosa, que ha terminado haciendo olvidar la deducción meramente matemática de las desigualdes de Heisenberg en el seno de la teoría cuántica.

En efecto, puede demostrarse (Bunge, 1967) que las únicas premisas necesarias para derivar las desigualdades de Heisenberg son:

a) Las relaciones de conmutación $p_{i} \cdot q_{j}-q_{i} \cdot p_{j}=(h / 2 \pi i) \delta_{i j}$, que son postulados de la teoría.

b) La definición de promedio cuántico para una variable dinámica $R$ cualquiera, que representa una cierta propiedad física de una entidad dada en un estado $\psi$, definido como $\langle R\rangle_{\psi}=[\psi, \widehat{R} \psi]$. Aquí $\widehat{R}$ es el operador cuántico asociado a la propiedad $R$ y $[. . . .$.$] son los corchetes de Dirac que simbolizan la$ operación producto escalar en el espacio de Hilbert.

c) La definición de desviación típica (o “dispersión standar”), una fórmula tomada de la estadística matemática cuyo enunciado es, para la misma magnitud genérica $R, \Delta_{\psi} R=\left\langle\left(R-\langle R\rangle_{\psi}\right)^{2}\right\rangle_{\psi}^{1 / 2}$.

d) Y, por último, la desigualdad de Schwarz, tomada del análisis matemático.

Queda claro, pues, que desde un punto de vista teórico las desigualdades de Heisenberg nada tienen que ver con observadores, mediciones o dispositivos experimentales, pese al sinfín de escritos en los que se instruye a los estudiantes afirmando justamente lo contrario.

\subsection{Resultados de la prueba}

Con el fin de explorar los errores conceptuales de los docentes en torno a este controvertido asunto, y antes de haber analizado los libros de texto en que los adquirieron, se entregó un cuestionario a veinticinco profesores del área de física y química de la educación secundaria y bachillerato en ejercicio en la provincia de Alicante, en sus respectivos centros y a comienzos del curso 2008-2009. La pregunta formulada era en su literalidad: "Elija entre las tres posibilidades siguientes de explicación sobre el significado de las desigualdades de Heisenberg, y si está en desacuerdo con todas ellas exponga la razón relatando cuantos detalles considere oportuno añadir", al objeto de que las respuestas fuesen lo más correctas y completas posible. Con ello se tenía el propósito de evitar que alguien juzgase innecesario agregar ideas o conceptos que no utiliza en las explicaciones a sus alumnos de enseñanzas medias. El resultado de la encuesta no resultó en absoluto alentador: ninguno de los encuestados ofreció una explicación mínimamente correcta.

\footnotetext{
${ }^{1}$ Las referencias que contienen información defectuosa o errónea, se indican en el contexto de este trabajo, aun cuando se citen junto
} con el resto de las referencias bibliográficas correctas. 
Las propuestas ofrecidas en el cuestionario podrían clasificarse en tres grupos, laxamente denominados como empirista, fenomenista y estocástica. Examinemos a continuación los rasgos generales de las tesis planteadas y la proporción en que se dio cada una de ellas.

a) Tesis Empirista: Las desigualdades de Heisenberg reflejan inexactitudes e incertidumbres experimentales debidas a la tosquedad del aparato de medición. Los micro-objetos, por consiguiente, poseen una posición y un momento precisos en todo instante, pero nosotros somos incapaces de determinarlos con exactitud a causa de la perturbación que el dispositivo de medida produce sobre el objeto observado. (Sostenida por 17 de los encuestados; $68 \%$ ).

Esta es una creencia que no por muy extendida resulta menos errónea (Gamow, 1965, 1980). Desde este punto de vista, es el bajo poder de resolución inherente a los dispositivos de medida -sistemas de medida al fin y al cabo- el responsable de que midamos generalmente intervalos de autovalores y no autovalores individuales (salvo cuando tales autovalores son discretos y están muy separados). Por ello, las desviaciones aleatorias en torno a la media de las medidas no carecen de causa, pues están ocasionadas por el aparato de medición.

Sin embargo, es fácil comprobar que las premisas de las que se deducen las fórmulas de Heisenberg no contienen supuesto alguno acerca de los dispositivos de medida, de modo que las dispersiones de autovalores existen incluso para entes cuánticos libres (Margenau, 1963). La "incertidumbre", en sentido estricto, es un estado mental que no aparece mencionado en postulado alguno de la teoría cuántica (en todo caso sería competencia de la psicología). Pero, por desgracia, en el contexto cuántico - a diferencia de las restantes ramas de la física- se suele utilizar este término para denotar los errores de medida de las dispersiones de la posición y el momento, $\varepsilon(\Delta q)$ y $\varepsilon(\Delta p)$.

Obviamente, la física cuántica no predice la forma y carácter de tales errores puesto que dependen de la técnica de medición.

b) Tesis Empirista: Las desigualdades de Heisenberg surgen de la imposibilidad de trazar un límite definido entre el observador y el objeto de observación (de lo que se infiere a menudo que el observable no existe con independencia del observador). En consecuencia, todo enunciado sobre un objeto debe incluir asimismo el procedimiento de observación, como ocurre en la teoría cuántica, donde todas sus fórmulas se refieren a situaciones experimentales. (Defendida en 2 respuestas; $8 \%$ ).

La doctrina fenomenista esbozada arriba mantiene que lo único realmente existente es una especie de trinidad indivisible constituida por el observador, sus instrumentos de medición y el objeto observado. La distinción entre estos tres componentes es ambigua y subjetiva, de manera que las dispersiones establecidas en las desigualdades de Heisenberg atañen a este conjunto tripartito y no a los microobjetos individualmente considerados, de los cuales ni siquiera puede decirse propiamente que existan al margen del acto de observación.

Quienes habían escogido esta opción parecían haber asumido plenamente la tesis fenomenista en un peculiar proceso de sustitución de una perspectiva epistemológica errónea por otra igualmente equivocada. Los afectados afirmaban - con razón- que esa era la posición ortodoxa de la llamada "escuela de Copenhagu" expuesta en numerosos textos al respecto (Bohr 1934, 1958; Frank 1938, 1946; Heisenberg, 1958), y no se percataban del burdo operacionalismo en que caían con ello. 
Basta con volver a examinar nuevamente las premisas en que se basan las desigualdades de Heisenberg, para comprobar que en sus postulados no aparece mención alguna a observadores ni a dispositivos medidores. Es más, las variables cuya dispersión se establece en estas fórmulas, corresponden exclusivamente al micro-objeto con independencia de su entorno (Bunge, 1973). Los enunciados fundamentales de la física cuántica son completamente generales (por la propia definición del término ?fundamentales?) e independientes de las técnicas de medición empleadas en cada situación experimental. Lógicamente, todos los protocolos experimentales contienen descripciones de aparatos y medidas, pero su intención es justamente garantizar el máximo grado de objetividad en la experiencia, no convertirse en tributarios de un subjetivismo idealista del todo improcedente. De hecho, no faltan los desarrollos de la teoría cuántica en los que la reducción del vector de estado se entiende como un proceso objetivo desde una concepción realista de la ciencia (Braginsky, 1977; Diosi, 1989; Ghirardi-Grassi-Pearle, 1990; Gissin-Percival, 1993; Pearle, 1969; Penrose, 1993; Percival, 1994; y muchos otros).

La tesis fenomenista, en fin, se sustenta en la doctrina filosófica según la cual carece de sentido hablar de la existencia de objetos en sí mismos, al margen de la actividad cognoscitiva de los sujetos que los perciben. Esta postura, que no encuentra asiento alguno en los postulados básicos de la teoría cuántica, se funda en una corriente de pensamiento que fue popular en las décadas de 1920 y 1930 -el positivismo lógico- pero que ya hace tiempo sucumbió a las críticas técnicas de los filósofos (Agassi, 1975; Bunge, 1967, 1973; Hempel, 1965; Popper, 1959).

c) Tesis Estocástica: Las dispersiones de Heisenberg son propiedades objetivas de las cosas en sí mismas - como la masa y la carga, por ejemplo - que indican el predominio del azar en el nivel microfísico. (Señalado en 6 respuestas; $24 \%$ ).

A la vista de lo discutido con anterioridad, esta opinión parecería ser la más verosímil (y no es extraño que algunos hayan relacionando, indebidamente, el comportamiento aleatorio de los entes cuánticos con las conductas caóticas derivadas de la dinámica de sistemas no lineales? 2 últimamente muy en boga). De acuerdo con ella, las fluctuaciones cuánticas se producirían tanto si hay observación como si no, agitando los micro-objetos en un zarandeo sin fin (Born, 1949; Landé, 1955).

Ahora bien, esta opinión contraviene las relaciones de conmutación que se encuentran en la base de las desigualdades de Heisenberg. Si nos imaginamos los objetos cuánticos como diminutos gránulos presa de interminables convulsiones que los llevan continuamente de aquí para allá, estaremos dando por sentado que en todo instante tienen autovalores bien definidos de la posición (y por ende también del momento) aunque sus violentas fluctuaciones nos impiden determinarlos. Tal presupuesto contradice abiertamente las relaciones de conmutación, las cuales demuestran con claridad lo contrario.

La tesis estocástica adolece del defecto implícito que ha resultado también fatal para las otras dos discutidas previamente, esto es, la suposición tácita de que los micro-objetos son entidades puntiformes y que, por lo tanto, tienen una posición definida en todo tiempo aun cuando nosotros por alguna razón seamos incapaces de determinarla. Pero resulta que esta hipótesis no pertenece a la teoría cuántica como tal; es un añadido espúreo ocasionado por la fuerza de nuestros hábitos de pensamiento clásico. Los objetos cuánticos no son partículas puntuales cuyas coordenadas de posición fluctúan tan abruptamente que sólo podemos hallar sus distribuciones, por la sencilla razón de que el concepto de ?coordenada de posición de una partícula? no existe en la teoría cuántica puesto que su referente físico no son

${ }^{2}$ Para más información sobre el caos determinista puede consultarse el magnífico manual: RAÑADA, A., (1990). Dinámica Clásica. Madrid: Alianza Editorial 
"partículas".

Las mal llamadas "autofunciones de posición" - deltas de Dirac - no son funciones en absoluto, por lo que difícilmente pueden proporcionar "autovalores de posición" de nade ${ }^{3}$ Esta es una de las diferencias cruciales entre la teoría cuántica y el caos no lineal (Alemañ, 1998): en esta última sí hay una función de posición bien definida cuya utilidad predictiva se pierde por el crecimiento desbocado del error en los datos iniciales, mientras que en la primera teoría tal función no existe. El operador de posición cuántico suministra coordenadas de campo (es decir, etiqueta puntos del espacio) no coordenadas de partícula (no localiza entidades físicas puntuales, como en la mecánica clásica). De hecho, la expresión "mecánica cuántica" es incorrecta, pues al no definir posiciones ni trayectorias (no podemos integrar la ecuación $d\langle x\rangle / d t=\langle p\rangle / m$ para obtener la trayectoria del objeto: como no hay posiciones, no hay trayectorias), no es realmente una mecánica.

Si bien los micro-objetos no son partículas, tampoco son ondas en la acepción clásica del término. Si fuesen partículas no podrían interferir consigo mismos en los experimentos de difracción con doble rendija, y si fuesen ondas bastaría un sólo impacto en la pantalla del experimento para obtener toda la figura de difracción (Feynman, 1986). La conclusión es que los objetos cuánticos son entes de una naturaleza física radicalmente nueva y distinta de los objetos clásicos, razón por la cual no cabe aplicar a aquéllos los mismos conceptos que inventamos para éstos. Tanto es así que, para evitar connotaciones debidas a la costumbre y asociaciones mentales inoportunas, han sido propuestos nuevas denominaciones para ellos, como "cuantones" o "particuanlas" (Lévy-Leblond, 1973)

\begin{tabular}{|l|c|c|c|c|}
\hline & $\begin{array}{c}\text { Tesis } \\
\text { Empirista }\end{array}$ & $\begin{array}{c}\text { Tesis } \\
\text { Fenomenista }\end{array}$ & $\begin{array}{c}\text { Tesis } \\
\text { Estocástica }\end{array}$ & $\begin{array}{c}\text { Desglose } \\
\text { según titulación }\end{array}$ \\
\hline Físicos & $5(20 \%)$ & $1(4 \%)$ & $4(16 \%)$ & $10(40 \%)$ \\
\hline Químicos & $11(84 \%)$ & $1(4 \%)$ & $3(12 \%)$ & $15(60 \%)$ \\
\hline Total & $17(68 \%)$ & $2(8 \%)$ & $6(24 \%)$ & $25(100 \%)$ \\
\hline
\end{tabular}

Tabla 1.1

El cuadro superior resume los resultados del trabajo en función de las opiniones defendidas y las titulaciones esgrimidas. Se observa una clara tendencia entre los químicos - que son mayoría en el grupo encuestadoa dar por válida la tesis empirista sin más, actitud probablemente imputable al uso sobre todo operativo que se hace de la teoría cuántica en la carrera de química. Entre los físicos las posturas se muestran mucho más igualadas. $\mathrm{Y}$ en conjunto, el porcentaje de quienes apoyan las ideas empiristas $(68 \%)$ supera con creces a los demás $(32 \%)$.

\subsection{Conclusiones y propuestas metodológicas.}

Las conclusiones definitivas que cabe extraer de este trabajo son las siguientes: en cuanto a la interpretación de las desigualdades de Heisenberg, es incorrecto entenderlas como perturbaciones debidas al acto de medición, como errores de la medida o como convulsiones inmanentes al movimiento de los micro-objetos. Los

${ }^{3}$ La situación es todavía peor en el caso de las teorías cuántico-relativistas, como las de Dirac o Kremer, en las que hay más de un "operador de posición". 
postulados de los que se deriva este teorema - que debería denominarse "teorema de incompatibilidad de operadores cuánticos"- no incorporan enunciados sobre experimentadores o dispositivos de medida, por lo que no resulta legítimo invocarlos después para su interpretación; no puede aflorar en un teorema un referente que no aparecía en los axiomas de partida. Es nuestro apego al modelo de la partícula puntual, con valores definidos de posición y velocidad en todo instante, el que nos arrastra a considerar las dispersiones en las distribuciones de autovalores como errores causados por alguna perturbación externa.

En definitiva, los cuantones - según el neologismo propuesto con anterioridad- son objetos extendidos en el espacio que carecen de figura o límites definidos, por lo que no pueden identificarse con una onda ni con un corpúsculo. Las desigualdades de Heisenberg reflejan así un aspecto clave de la propia naturaleza de las micro-entidades materiales, que ninguna relación guarda con errores de medida o incertidumbres de observadores. Y tampoco justifican una postura filosófica cercana al indeterminismo radical, ya que ellas mismas constituyen una ley de la naturaleza, si bien es cierto que se apartan del determinismo clásico (Bunge, 1959).

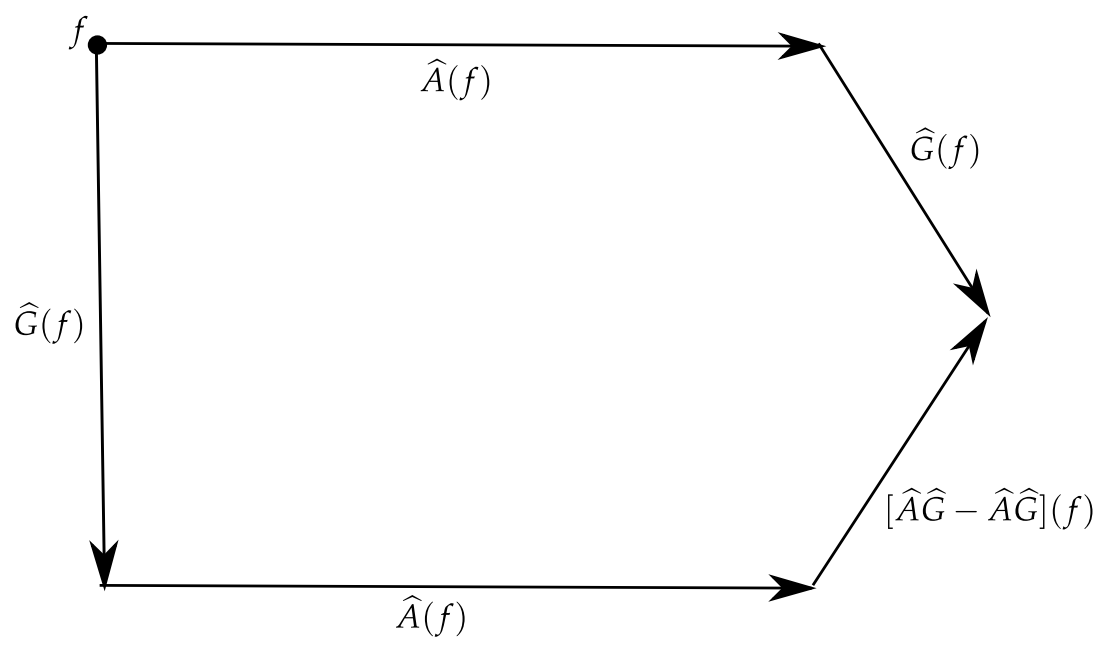

Figura 1.1

A modo de ilustración didáctica, el conmutador de dos operadores aplicados a una función $f$ puede entenderse como el proceso de construcción de un cuadrilátero por transporte sucesivo de dicha función a lo largo de dos caminos alternativos fijados por la aplicación a $f$ de ambos operadores en orden inverso (véase la figura (1.1). Sobre un espacio funcional abstracto en el cual imaginamos la función $f$ representada como un punto, aplicamos sobre ella el operador $\widehat{A}$ y la transformamos en otro "punto", el cual puede unirse con el origen mediante un vector al que llamamos $\widehat{A}(f)$. Aplicamos a continuación el operador $\widehat{G}$ y obtenemos el vector $\widehat{G}(f)$. Ahora partamos de nuevo desde $f$ aplicando en esta ocasión primero el operador $\widehat{G}$ y después el $\widehat{A}$. Los puntos finales a que conducen respectivamente las secuencias $\widehat{A} \widehat{G}$ y $\widehat{A} \widehat{G}$ son distintos: entre ellos existe una separación expresable mediante un vector $[\widehat{A} \widehat{G}-\widehat{A} \widehat{G}](f)$.

Si ambos operadores hubiesen sido conmutables, de modo que el orden de su aplicación careciese de importancia, los dos pares de vectores hubiesen terminado coincidiendo en el mismo punto; es decir, hubiesen formado un polígono cerrado. La no conmutatividad de los mismos se expresa por la falta de cierre de dicho polígono. Y el vector necesario para su clausura es precisamente el dado por $[\widehat{A} \widehat{G}-\widehat{A} \widehat{G}](f)$

Este efecto sobre un espacio funcional es equivalente al que ocurre sobre un espacio afín con curvatura intrínseca no nula. En ese caso se sabe que el transporte paralelo de un cierto vector a lo largo de una trayectoria 
cerrada depende del camino escogido para trasladarlo $(1.2)$.

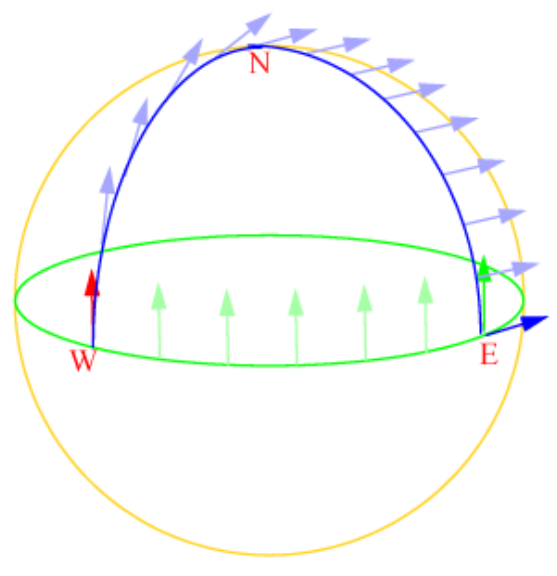

Figura 1.2

En otras palabras, el transporte paralelo de un vector sobre un variedad con curvatura intrínseca distinta de cero, depende del camino elegido. Resulta fácil comprobar que partiendo del punto $W$ sobre la superficie de una esfera con un vector que paralelo a ese meridiano, si la trasladamos paralelamente a sí misma pasando por el polo $N$ hasta el punto $E$, su orientación final difiere de la obtenida al trasladar ese mismo vector a lo largo del ecuador de la esfera directamente de $W$ a $E$. Lo que en el caso de las funciones abstractas se representa mediante un vector de cierre $[\widehat{A} \widehat{G}-\widehat{A} \widehat{G}](f)$, aquí se percibe de forma mucho más visual como la diferencia de orientaciones entre ambos vectores, debida a la curvatura de la variedad sobre la cual se desplazan.

Los docentes harían bien en reflexionar sobre las contradicciones entre lo que creen saber sobre ciertas teorías y lo que realmente éstas afirman. De ahí el falso cambio conceptual, pues se cree comprender una nueva teoría física cuando en realidad se reinterpretan sus conceptos de acuerdo con las ideas antiguas (resistencia al cambio conceptual). Un examen atento de las premisas de la física cuántica desde una perspectiva realista y objetiva - la única compatible con el genuino método científico- es el mejor antídoto contra las concepciones sesgadas por los prejuicios operacionalistas e idealistas que tan difundidos se hallan.

En este contexto, el prejuicio operacionalista se refiere al rechazo de cualquier concepto físico cuya interpretación no se halle directamente ligada a un procedimiento de medida específico. Una consideración más detenida nos revela que puede haber muy diversas maneras de medir una misma magnitud física (tantas como dispositivos de medida y como técnicas para emplear cada uno de ellos), razón por la cual no se puede establecer una relación necesariamente biunívoca entre el significado de un concepto físico y las operaciones experimentales utilizadas para asociarle una medida concreta.

En relación con los alumnos, son muchos los docentes en cuya opinión estos contendidos son demasiado complejos y profundos para ser asimilados por alumnos preuniversitarios, y tal vez no les falte razón. Sin embargo, no sería honesto dejarles marchar de las aulas con la idea de que en la microfísica las cosas funcionan simplemente como una miniatura del mundo clásico. Debería caracterizarse bien las propiedades clásicas de las ondas y de las partículas para luego contraponerlas con las propiedades distintivas de los cuantones, propiedades que les hacen ser algo tajantemente distinto de todo lo conocido antes. Ya que quizás no podamos explicarles con toda claridad lo que los cuantones son porque en el fondo ni siquiera nosotros lo sabemos sí deberíamos hacerles entender lo que no son. De esta forma les prepararíamos, a la vez que 
nos preparamos a nosotros mismo, para construir nuestro propio aprendizaje en uno de los campos más complicados y fascinantes de la física del siglo XX.

\section{Bibliografía}

[1] Agassi, J., (1975). Science in Flux, Dordrecht-Boston: Reidel.

[2] Aleman Berenguer, R. A., (1985). Grandes Metáforas de la Física. Madrid: Ed. Celeste - MEC.

[3] Alonso, M.-Finn, E., (1971). Física Vol. III. México: fondo educ. Interamericano.

[4] Baird, J.R., (1988). Teachers in science education, en fensham, p.j. (ed.), Development and dilemmas in science education. (the falmer press: londres).

[5] Bohr, N., (1934). Atomic theory and the description of nature. Cambridge: cam. Univ. Press.

[6] Bohr, N., (1958). Atomic physics and human knowledge. Nueva york: wiley.

[7] Born, M:; (1949). Natural philosophy of cause and chance. Oxford: clarendon press.

[8] Braginsky, V.B., (1977). The detection of gravitational waves and non distributive measurements en topics in theoretical and experimental gravitational physics. Londres, Plenum Press.

[9] Bunge, M., (1959). Causality, Cambridge (Mass.). Harvard Univ. Press.

[10] Bunge, M., (1967). Foundations of physics. Berlin-Heildelberg-Nueva York. Springer- Verlag.

[11] Bunge, M., (1970). Canadian journal of physics, 48, p. 1410

[12] Bunge, M., (1973). Philosphy of physics. Dordrecht (Holanda). Reidel.

[13] Dicke, R.-Wittke, J.P., (1975). Introducción a la mecánica cuántica. Zaragoza. Ed. Librería general.

[14] Diosi, L., (1989). Models for universal reduction of macroscopic quantum fluctuations. Physics Review, a40, p. 1165.

[15] Feynman, R., (1986). El carácter de la ley física. Barcelona. Orbis.

[16] Frank, P., (1938). Interpretations and misinterpretations of modern physics. Paris: Hermann.

[17] Frank, P., (1946). Foundations of physics. Univ. of Chicago Press.

[18] Gamow, G., (1965). Thirty years that shook physics. Double day \& co. (n.y.)

[19] Gamow, G., (1980). Biografía de la física. Alianza editorial (madrid)

[20] Gassiorowicz, S.,(1974). Quantum physics. Nueva York. Wiley

[21] Ghirardi, G.C.- Grassi, R.- Pearle, P., (1990). Relativistic dinamical reduction models: general framework and examples. Foundations of Physics, 20, p. 1271.

[22] Gisin, N.- Percival, I.C., (1993). Stochastic wave equations versus parallel world components. Physics Letters, a 175, p. 144.

[23] Gunstone, R.F., (1988). Learners in science education. En Fensham, p.j. (ed.), Development and dilemmas in science education. The Falmer Press. Londres

[24] Heisenberg, W., (1925). Z. f. Phys., 33, p. 879.

[25] Heisenberg, W., (1927). Z. f. Phys., 43, p. 172.

[26] Heisenberg, W., (1930). The physical principles of the quantum theory. Chicago: University of Chicago Press.

[27] Heisenberg, W., (1958). Physics and philosophy. Nueva york: Harper \& Brothers.

[28] Hempel, C.G., (1965). Aspects of scientific explanation. Nueva York: Free Press.

[29] Lande, A., (1955). Foundations of quantum theory. New Haven: Yale Univ. Press. 
[30] Levine, Y., (1977), Química cuántica. Madrid: Ed. Ac.

[31] Lévy-leblond, J.M., (1973). Bulletin de la société française de physique, 14, Encart Pedagogique I, Paris.

[32] Margenau, H., (1963). Annals of phys., 23, p. 469

[33] Osborne, R.J. - wittrock, m.c., (1983).Learning science: a generative process. Science education, 67(4), pp. 489-508.

[34] Pearle, P., (1976). Reduction of stae-vector by nonlinear scroedinger equation. Physicsreview, d13, P. 857.

[35] Penrose, R., (1993). Gravity and quantum mechanics. Proceedings of the 30th International Conference on General Relativity. Bristol: Inst. of Phys. Publications.

[36] Percival, I.C., (1994). Primary state diffusion. Londres: Proceedings of the Royal Society.

[37] Popper, K., (1959). The logic of scientific discovery. Londres: Hutchinson.

[38] Wichman, E.H., (1991). Física cuántica, Barcelona: Reverté. 